\title{
Development of a novel customized cutting and rotating template for Bernese periacetabular osteotomy
}

Xuyi Wang ${ }^{1,2 \dagger}$, Shixian Liu ${ }^{3+}$, Jianping Peng ${ }^{2}$, Zhonglian Zhu' ${ }^{1}$ Linlin Zhang ${ }^{4}$, Jianzhong Guan ${ }^{1 *}$ and Xiaodong Chen ${ }^{2^{*}}$

\begin{abstract}
Background: Bernese periacetabular osteotomy (PAO) has been shown to be applicable as a hip-preserving technique for the treatment of developmental dysplasia of the hip (DDH). The approach could be designed preoperatively using various types of reverse-engineering software and finite element analysis, but how to implement it in the actual PAO remains a challenge. This study examines and evaluates a solution to achieve higher accuracy when performing a PAO.
\end{abstract}

Methods: A patient-specific cutting and rotating template was predesigned through computer-aided design (CAD) with three-dimensional (3D) modeling programs. The templates were then reproduced with rapid prototyping (RP) technology and used in the actual PAO. Finally, the clinical and radiographic effects were assessed and compared between the newly developed PAO and conventional PAO groups.

Results: The customized cutting template fit well with the bone surface and served as a guide for surgeons as they slid the osteotome to the precise location that had been determined prior to surgery. A very similar acetabular fragment was reproduced, and no major complications occurred when performing the osteotomy along the edge of the cutting template. The acetabular fragment was then corrected to the predetermined position through one-off manipulation with the customized rotating template. The final position of the acetabular fragment in the new developed PAO group was highly consistent with the planned position, and the postoperative morphological parameters were consistent with the preoperative planned data compared to the conventional PAO group. The duration of the operation and the number of irradiation decreased significantly. The Harris hip score (HHS) and visual analogue scale (VAS) score improved significantly with the use of the new developed PAO.

Conclusions: We demonstrate that our system, which was based on CAD-RP technology, is feasible and could realize the predicted results accurately during the actual PAO.

Keywords: Periacetabular osteotomy, Developmental dysplasia of the hip, 3D printing technology, Customized template

\footnotetext{
* Correspondence: tanglijunwxy@sina.com; chenxdmd@163.com

${ }^{+}$Xuyi Wang and Shixian Liu contributed equally

${ }^{1}$ Department of Orthopaedics, The First Affiliated Hospital of Bengbu Medical

College, Bengbu, Anhui, China

${ }^{2}$ Department of Orthopaedics, Xinhua Hospital affiliated to Shanghai

Jiaotong University School of Medicine, Shanghai, China

Full list of author information is available at the end of the article
}

(c) The Author(s). 2019 Open Access This article is distributed under the terms of the Creative Commons Attribution 4.0 International License (http://creativecommons.org/licenses/by/4.0/), which permits unrestricted use, distribution, and

reproduction in any medium, provided you give appropriate credit to the original author(s) and the source, provide a link to the Creative Commons license, and indicate if changes were made. The Creative Commons Public Domain Dedication waiver (http://creativecommons.org/publicdomain/zero/1.0/) applies to the data made available in this article, unless otherwise stated. 


\section{Background}

Developmental dysplasia of the hip (DDH) is a complex, three-dimensional (3D) deformity characterized by variances in the shape, size, and orientation of the acetabulum and/or proximal femur [1-3]. The prevalence of DDH varies significantly among different racial groups, from $0.1 \%$ in Hong Kong, China, to $75 \%$ in Greece and Italy [4]. Acetabular dysplasia is a primary cause of secondary osteoarthritis of the hip in adolescents and young adults [5-7]. Early and prompt surgical intervention for $\mathrm{DDH}$ is one of the most effective ways to prevent or delay further degeneration of the hip joint. Many surgical procedures have been reported to improve joint coverage and femoral head-acetabular congruency [8-10]. Among these, Bernese periacetabular osteotomy (PAO), proposed by Ganz et al. [11], because of its advantages such as the dorsal pillar remains mechanically intact without compromising the dimensions of the birth canal and allowing excessive potential for acetabular reorientation, has become the preferred hip-preserving surgery for treating DDH at many centers worldwide. Various studies have reported good to excellent PAO results in both clinical and radiological observations with both mid- and longterm follow-ups [12-14].

Over the last few years, with the growing use of computer technology in medical imaging, computer-aided virtual surgery based on a 3D model has been widely used in clinical settings $[15,16]$. The reconstructed $3 \mathrm{D}$ model can be evaluated from any perspective. Surgeons can identify the location and severity of an acetabular deficiency preoperatively and apply finite element analysis to determine the optimal corrections of the acetabular fragment [17, 18]. The correction of the acetabular fragment relies largely on the surgeon's clinical experience in conventional PAO. However, accurately implementing the preoperative planning during the actual procedure is still a challenge for surgeons. Some studies have applied computer-assisted navigation $[19,20]$ to improve the accuracy of surgery. However, these techniques have several disadvantages, including the need for expensive equipment, a long learning curve, a time-consuming process, and errors that occur due to shifts in the registration frame [21]. Another method for transferring the surgical plan to the operating field involves the use of various customized templates that are designed with computer-aided design (CAD) technology and manufactured with rapid prototyping (RP) technology [22, 23]. Compared with conventional surgery, the customized template has obvious advantages in simulating preoperative procedures, which are helpful in determining the feasibility of surgery and selecting the implant type, size, and position in a complex total hip replacement. CAD-RP technology has been widely used in other orthopedics procedures.
However, there have been no studies on the use of this technology for PAO [24].

Based on the application of this technology in other types of surgery, we developed a computer-assisted surgical planning and 3D model-based cutting and rotating template for PAO in 2014. The aim of the present study is to describe the procedure, evaluate the feasibility and accuracy of this method, and compare our short-term results with those of conventional PAO.

\section{Methods}

\section{Patients and data acquisition}

The study protocol was approved by our institutional review board, and informed consent was obtained from all patients. All investigations conformed with the ethical principles of research.

From May through December 2017, we examined 20 hips in 20 consecutive subjects who had experienced hip pain for at least 6 months and were diagnosed with acetabular dysplasia by physical examinations and roentgenography. Of these, 8 hips in 8 cases (average age, $26 \pm 8$ years; range, 16 to 38 years) were treated with PAO using the customized cutting and rotating template, while the remaining 12 hips in 12 cases (average age, $25 \pm 5$ years; range, 18 to 35 years) were treated with conventional PAO. All cases selected for the study were all Crowe I or Crowe II [25] and $\leq$ grade 1 according to the Tönnis grading system. Each eligible patient completed a baseline evaluation comprising questions about pain severity and functional status and underwent a physical examination. The patients who had hip surgery history (such as open reduction), other hip deformities (such as Legg-Calvé-Perthes), proximal femoral deformity (such as coxa varus and/or valgus), Crowe III IV, and Tönnis grade of 2 were excluded. Participants who were referred to the outpatient center on odd and even days were assigned to the conventional PAO or the new developed PAO group, respectively. The subjects' clinical data are summarized in Table 1.

The preoperative and postoperative $\mathrm{CT}$ imaging data (from the top of the sacrum down to the level of the lesser trochanters) of each patient were obtained using the Siemens 64 channel scanner (Siemens Healthcare, Munich, Germany). Helical scanning was conducted at $120 \mathrm{kVp}$ and $300 \mathrm{mAs}, 512 \times 512$ matrix, 0.7539 pitch, 300 to $400 \mathrm{~mm}$ field of view, and $0.75 \mathrm{~mm}$ axial slice thickness. The patients were placed in the supine position, with their hips and knees fully extended, patellae pointing straight up, and the tiptoe stabilized at $15^{\circ}$ of internal rotation. Care was taken to ensure that the midline of the body was aligned with the midline of the scanning table. All CT data were converted to the digital 
Table 1 The clinical data of the subjects included in this study

\begin{tabular}{llll}
\hline & New developed PAO & Conventional PAO & $p$ value \\
\hline Numbers (hips) & $8(8)$ & 12 (12) & $7 / 5$ \\
Female/Male & $5 / 3$ & $25 \pm 5(18$ to 35$)$ \\
Ages (years) & $26 \pm 8(16$ to 38$)$ & $22 \pm 2(18$ to 24$)$ & $12 \pm 5(7$ to 24$)$ \\
BMI $\left(\mathrm{kg} / \mathrm{m}^{2}\right)$ & $21 \pm 2(19$ to 23$)$ & $67 \pm 5(61$ to 78$)$ & 0.8549 \\
Durations (months) & $13 \pm 5(6$ to 20$)$ & $3.0 \pm 0.4(2.6$ to 3.6$)$ & 0.07 \\
Harris hip score & $66 \pm 5(61$ to 77$)$ & & 0.4118 \\
Visual analogue score & $3.2 \pm 0.6(2.5$ to 4.5$)$ &
\end{tabular}

Values are expressed as mean \pm standard deviation with range in parentheses

imaging and communications in medicine (DICOM) format and imported into a personal computer to generate the $3 \mathrm{D}$ model of the pelvis.

\section{Preoperative planning}

The 3D model of the pelvis was reconstructed using the Imageware 13.1 reverse-engineering software program (SensAble Technologies, Woburn, MA, USA). For each patient, the severity and direction of acetabular deficiency were evaluated by measuring the anatomical morphological data, including the lateral center-edge angle (LCEA), the anterior center-edge angle (ACEA), the acetabular anteversion angle (AAVA), the anterior acetabular sector angle (AASA), and the posterior acetabular sector angle (PASA) (Fig. 1a-e, respectively). As the position of the pelvis (i.e., pelvic tilt and pelvic rotation) has a considerable effect on the measurement of the acetabular angle, we defined the anterior pelvic plane, which involves the bilateral anterior superior iliac spines and the pubic tubercles, as the specific anatomical reference plane (the standard coronal plane) to correct the pelvic position [15]. Based on these preoperative data, we planned the rotation angle and direction of the free osteotomized fragment.

Based on Ganz's description [11], we performed a virtual PAO on the reconstructed 3D model by defining the corresponding cutting plane (Fig. 2a). Cutting plane 1 was defined as a plane perpendicular to the superior ramus of the pubis and $1 \mathrm{~cm}$ medial to the iliopubic eminence. Cutting plane 2 was defined as a plane from the anterior of the ischial body towards the ischial spine along the infracotyloid groove. Cutting plane 3 was defined as a plane between the anterior superior iliac spine and the anterior inferior iliac spine towards the greater sciatic notch. Cutting plane 4 was at an angle of $25^{\circ}-30^{\circ}$ anteversion to the standard coronal plane with a distance of $1.0-1.5 \mathrm{~cm}$ from the greater sciatic notch and connecting planes 2 and 3 to form an angle of $110^{\circ}-120^{\circ}$ relative to plane 3 .

An acetabular fragment almost identical to the actual PAO was reproduced through a series of periacetabular osteotomies along the margin of 4 cutting planes (Fig. 2b). Then, the rotation of the acetabular fragment was simulated anterolaterally (Fig. 2c). The degree and direction of the fragment were related to the severity of acetabular dysplasia and varied considerably among patients. After the above procedures were completed, a closing-wedge gap between the fragment and the residual pelvis was formed.

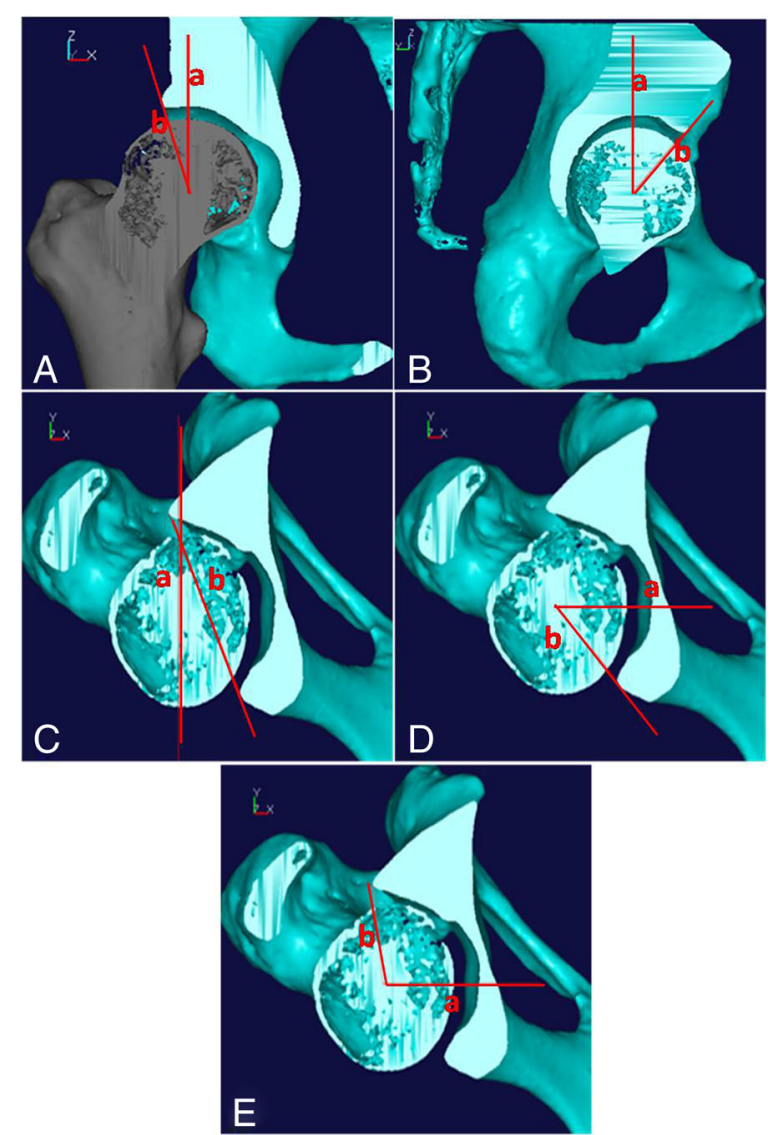

Fig. 1 The measurement methods of acetabular angle on 3D model. a The coronal image passing through the center of the femoral head, the intersection angle represent LCEA. $\mathbf{b}$ The sagittal image passing through the center of the femoral head, the intersection angle represent ACEA. c-e The axial image passing through the center of the femoral head, the intersection angle represent AAVA (c), AASA (d), and PASA (e) 

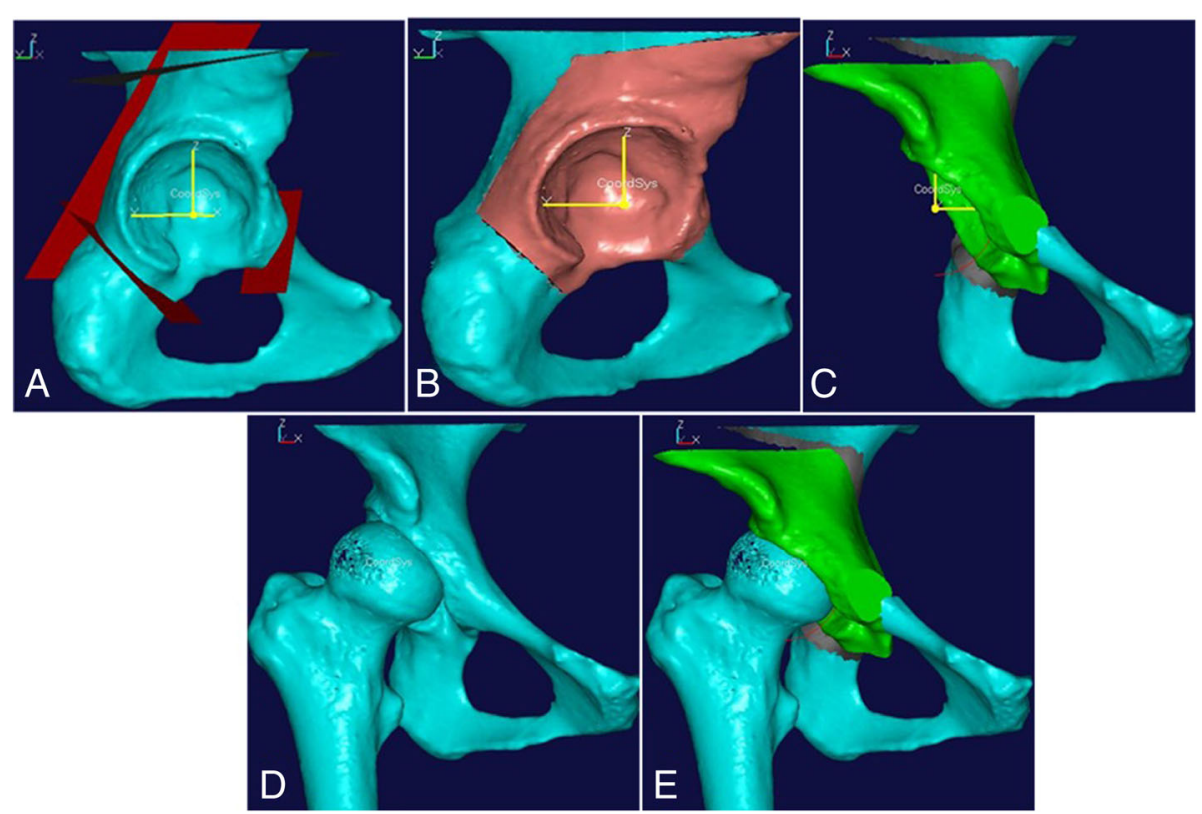

Fig. 2 The virtual PAO was performed on the reconstructed pelvic 3D model and show the effect chart between pre and post virtual PAO. a The defined cutting plane according to the osteotomy method described by Ganz. b The free acetabular fragment after performing virtual PAO. c The correct position of the acetabular fragment based on the comparison between the preoperative acetabular angle and the normal data. $\mathbf{d}$, e The comparison between pre and post virtual PAO shows that the femoral head coverage was improved

\section{Design and fabrication of the customized cutting and rotating template}

To reproduce the preoperative plan during surgery, a patient-specific cutting and rotating template was designed according to the preoperative simulation using Imageware 13.1. The details of the design process have been described elsewhere [16, 23, 26]. First, we extracted digital information regarding the free osteotomized fragment formed by the virtual PAO as described above. An appropriately sized block with two or three holes $(\phi 2.0 \mathrm{~mm})$ was placed on the surface of the acetabular quadrilateral body

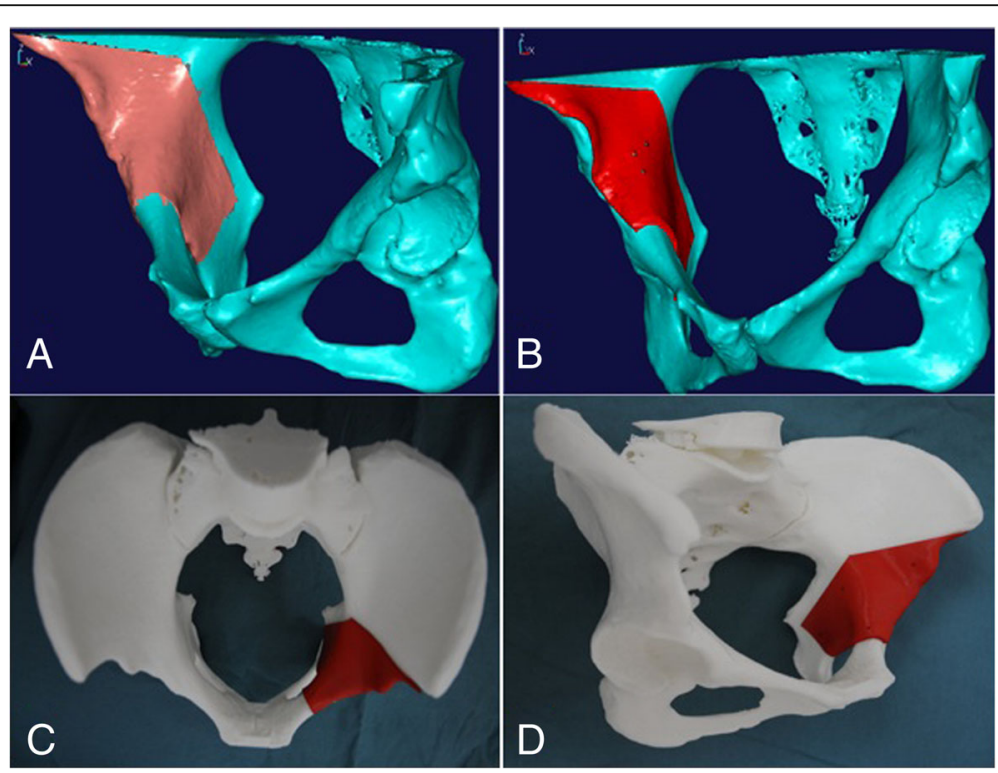

Fig. 3 The design process and entity generation of the cutting template. a The preoperative planned acetabular fragment. $\mathbf{b}$ The predesigned patient-specific cutting template matched with the contour the planned acetabular fragment through computer-aided design technology. $\mathbf{c}$, $\mathbf{d}$ The entity generation of the customized cutting template through rapid prototyping technology 
to be exposed during surgery. The holes were used to fix the cutting guide to the pelvis (Fig. 3a, b). Similarly, after correcting the fragment to the preoperative designed position, a unique block was reconstructed that filled the gap between the fragment and the residual pelvis (Fig. 4a, b). Second, the standard template library (STL) format data of the template were imported into FreeForm ${ }^{\circ}$ Modeling $^{\mathrm{Tm}}$ version10.0 (SensAble Technologies, Woburn, MA, USA), which is another commercial 3D modeling program. After the computer-aided design of the customized template was completed, it was manufactured with medical-grade plastic materials (polyamide 12) on a plastic laser sintering machine (Formiga P100; Electro Optical Systems GmbH, Munich, Germany) (Figs. 3c, d and 4c).

\section{Surgical procedure}

The surgical procedure has been described in detail by Ganz et al. [11]. A modified Smith-Peterson approach $(\sim 12 \mathrm{~cm})$ was performed by one senior surgeon (Chen Xiaodong) in all cases. The lateral femoral cutaneous nerve was exposed bluntly in the gap between the sartorius and tensor fascia lata. The anterior superior iliac spine (ASIS) was osteotomized with the inguinal ligament; the sartorius muscle and lateral femoral cutaneous nerve were retracted medially. The inner plate of the ilium and quadrilateral body were dissected subperiosteally, and the patient-specific cutting template was placed on the inside surface of the pelvis. After confirming that the template fit closely to the bone surface and no visible gap existed, the template was fixed to the pelvis with a 2.0-mm Kirschner wire. Osteotomies of the superior ramus of the pubis, ischium, ilium, and posterior acetabulum were performed in sequence along the template edge mounted on the quadrilateral surface (Fig. 5a, b). After four periacetabular osteotomies and a controlled fracture, the completely mobile acetabular fragment was obtained. The cutting template was removed, and the fragment was rotated anterolaterally with the help of two Schanze screws. The rotating template was placed into the gap between the acetabular fragment and the pelvis and fine-tuned to ensure that the template fit properly with the acetabular fragment and pelvis (Fig. 5c, d). The reduced position of the acetabular fragment was verified by image intensifier to ensure that femoral head coverage was improved, and the motion of the hip was checked to ensure that there was no femoroacetabular impingement. The corrected acetabular fragment was fixed with three cortical screws after the rotating template was removed, and the ASIS osteotomized fragment was fixed in situ with a $3.5 \mathrm{~mm}$ cortical screw. The duration of operation $(\mathrm{min})$, bleeding volume $(\mathrm{ml})$, and irradiation times (NO) were recorded for both groups. Patients were hospitalized for an average of 8.5 days (range, 7 to 12 days).

\section{Postoperative assessment}

One week after PAO, all subjects underwent X-ray examinations and $\mathrm{CT}$ scans. The acetabular angles (LCEA, ACEA, AAVA, AASA, PASA) were measured on the reconstructed $3 \mathrm{D}$ model of the pelvis. The accuracy of the customized template was assessed by comparing the deviation of the planned and postoperative acetabular angles in the two groups. In addition, the preoperative planned and postoperative $3 \mathrm{D}$ pelvic models of the new developed PAO group were superimposed to evaluate the congruence of the acetabular fragment from the geometry. The function of the hip joint was assessed using the Harris hip score (HHS). Pain of the hip joint at the time of the last follow-up was assessed with visual analogue scale (VAS) after the patient had walked for 30 to $60 \mathrm{~min}$. All subjects were asked to come to our department for regular examinations after PAO

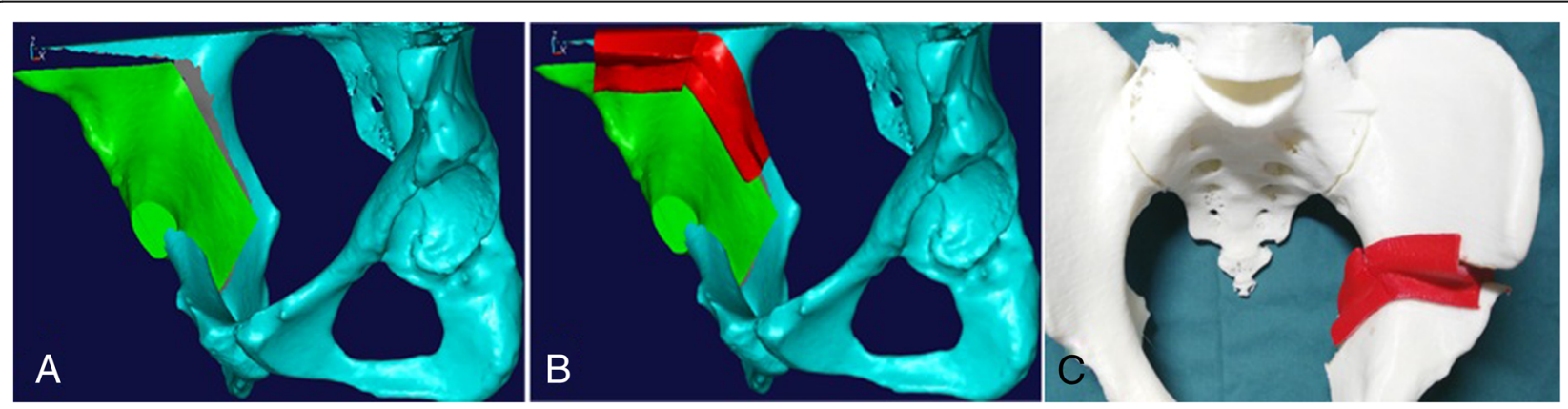

Fig. 4 The design process and entity generation of the rotating template. a The preoperative planned position of the acetabular fragment. $\mathbf{b}$ The predesigned patient-specific rotating template which filled the gap between the fragment and residual pelvis through computer-aided design technology. c The entity generation of the customized rotating template through rapid prototyping technology 


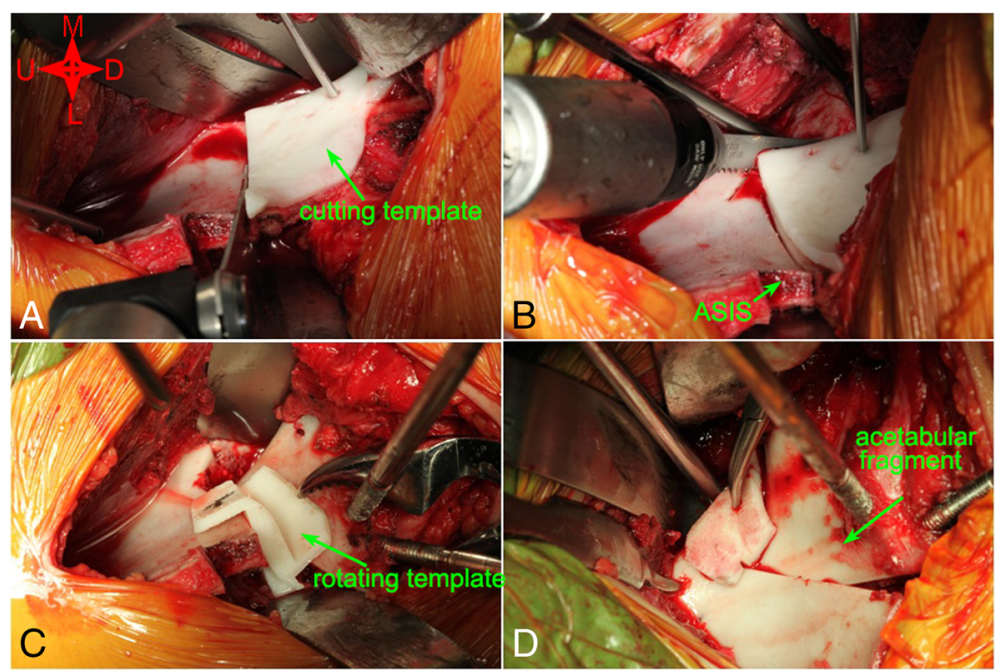

Fig. 5 Application of the patient-specific cutting and rotating template for PAO. a, b Fixed the patient-specific cutting template on the inside surface of the pelvis and cut off the superior ramus of the pubis, ischium, ilium, and posterior acetabulum in sequence along the template edge. $\mathbf{c}$, $\mathbf{d}$ Rotated the fragment anterolaterally and placed the rotating template into the gap between the acetabular fragment and residual pelvis and positioned the acetabular fragment at a unique location in three-dimensional space. (M: medial, L: lateral, U: up, D: down, ASIS: anterior superior iliac spine)

at 1 month, 3 months, 6 months, and 1 year. The HHS and VAS score at the last follow-up were recorded, and the hip joint was X-rayed to evaluate recovery. Improvement at the last follow-up and the preoperative HHS and VAS score of the two groups were compared to evaluate the clinical outcomes of the new developed PAO.

\section{Statistics}

Body mass index, duration of symptoms, and HHS and VAS score of the two groups were compared using Student's $t$ test. The duration of surgery, bleeding volume, and HHS and VAS score at the last follow-up were also compared using Student's $t$ test. Deviations of the LCEA, ACEA, AAVA, AASA, and PASA were calculated using Formula*. The deviations between the two groups were compared using Student's $t$ test. The normal distribution was estimated, and the results showed a good fit. Homogeneous variance was estimated by the $F$ test, and the $F$ values were greater than 0.05 . Statistical analyses were conducted using SPSS (version 15.0). Significance was determined at a $p$ value of $<0.05$.

Formula $*$ : Deviation $(\%)=\mid$ (postoperative value-planned value)

$$
\text { /planned value } \mid \times 100
$$

\section{Results}

There were no major complications, including posterior column fracture or intraarticular osteotomy, with either surgical procedure. The average duration of the operation and the number of irradiations required in the new developed PAO group were $102 \pm 7 \mathrm{~min}$ and $4 \pm 1$, while those in the conventional PAO group were $117 \pm 19 \mathrm{~min}$ and $7 \pm 2$, respectively. The differences between groups were statistically significant $(p<0.01)$. The bleeding volume in the new developed PAO group was higher than that in the conventional PAO group $(695 \pm 119 \mathrm{ml}$ vs $620 \pm 45 \mathrm{ml})$, but the difference was not statistically significant $(p=0.0617)$ (Table 2).

No subjects were lost to follow-up, and the mean follow-up was 12 months (range, 10 to 14 months). Preoperatively, the HHS of the two groups were $66 \pm 5$ (new developed PAO group) and $67 \pm 5$ (conventional PAO group). The VAS scores of the two groups were $3.2 \pm 0.6$ (new developed PAO group) and $3.0 \pm 0.4$ (conventional PAO group). The differences in the preoperative HHS and VAS score between the two groups were not statistically significant $(p=0.4118$ and 0.1586 , respectively). At the time of the last follow-up, the HHS had improved to $94 \pm 2$ in the new developed PAO group and $91 \pm 1$ in the conventional PAO group. The VAS score had improved to $1.0 \pm 0.8$ in the new developed PAO group and $1.3 \pm 0.9$ in the conventional PAO group. The HHS and VAS score improved significantly in the new developed PAO group compared to those in the conventional PAO group $(p<0.05)$ (Table 2$)$.

Intraoperative roentgenograms and postoperative CT scanning demonstrated that femoral head coverage had improved for all subjects, and no acetabular retroversion appeared. When the acetabulum is retroverted, the anterior 
Table 2 The clinical assessment of new developed PAO and conventional PAO

\begin{tabular}{|c|c|c|c|}
\hline Parameters & New developed PAO & Conventional PAO & $p$ value \\
\hline Duration of operation (min) & $102 \pm 7$ (92 to 110$)$ & $117 \pm 19$ (93 to 148$)$ & $<0.01$ \\
\hline Bleeding volume (ml) & $695 \pm 119$ (623 to 833$)$ & $545 \pm 81$ (415 to 710$)$ & 0.0617 \\
\hline Irradiation times (NO) & $4 \pm 1$ (3 to 6$)$ & $7 \pm 2(5$ to 10$)$ & $<0.01$ \\
\hline \multicolumn{4}{|l|}{ Harris hip score } \\
\hline Preoperative & $66 \pm 5$ (61 to 77$)$ & $67 \pm 5(61$ to 78$)$ & 0.4118 \\
\hline Last follow-up & $94 \pm 2$ (92 to 97$)$ & $91 \pm 1$ (90 to 94$)$ & $<0.01$ \\
\hline Improvement & $27 \pm 4$ (19 to 34$)$ & $24 \pm 5$ (12 to 30$)$ & $<0.01$ \\
\hline \multicolumn{4}{|l|}{ Visual analogue scale } \\
\hline Preoperative & $3.2 \pm 0.6$ (2.5 to 4.5$)$ & $3.0 \pm 0.4$ (2.6 to 3.6$)$ & 0.1586 \\
\hline Last follow-up & $1.0 \pm 0.8$ (0 to 2.6$)$ & $1.3 \pm 0.9(0$ to 2.8$)$ & 0.2531 \\
\hline Improvement & $2.1 \pm 1.0(0.2$ to 3.8$)$ & $1.7 \pm 1.1(0$ to 2.9$)$ & $<0.01$ \\
\hline
\end{tabular}

Values are expressed as mean \pm standard deviation with range in parentheses

wall lateralizes, and a "cross-over" sign is apparent with medialization of the superoposterior acetabular wall. The customized cutting template fit perfectly with the bone surface, and there was no visible gap in any of the eight patients. An acetabular fragment almost identical in shape and size to the planned one was reproduced by performing a series of periacetabular osteotomies along the margin of the cutting template. The acetabular fragment was then corrected to its planned position with the help of the rotating template. By superimposing the postoperative and preoperative planned $3 \mathrm{D}$ models of the pelvis, the final position of the acetabular fragment was confirmed to be highly consistent with the planned position (Fig. 6), and the postoperative morphological parameters were quite consistent with the preoperative planned data in the new developed PAO group (Table 3).

In the new developed PAO group, the preoperative LCEA, ACEA, AAVA, AASA, and PASA were $10^{\circ} \pm$ $5^{\circ}, 33^{\circ} \pm 7^{\circ}, 23^{\circ} \pm 2^{\circ}, 42^{\circ} \pm 4^{\circ}$, and $88^{\circ} \pm 3^{\circ}$, respectively, which were corrected to $30^{\circ} \pm 2^{\circ}, 53^{\circ} \pm 3^{\circ}, 20^{\circ} \pm 1^{\circ}$, $59^{\circ} \pm 2^{\circ}$, and $85^{\circ} \pm 3^{\circ}$, respectively, in the virtual PAO. The final values of these angles after the actual PAO were $31^{\circ} \pm 2^{\circ}, 54^{\circ} \pm 2^{\circ}, 19^{\circ} \pm 1^{\circ}, 58^{\circ} \pm 2^{\circ}$, and $85^{\circ} \pm 3^{\circ}$, respectively. The deviations between the angles were $4^{\circ} \pm 1^{\circ}, 3^{\circ} \pm 3^{\circ}, 4^{\circ} \pm 3^{\circ}, 3^{\circ} \pm 2^{\circ}$, and $1^{\circ} \pm 1^{\circ}$, respectively. In the conventional PAO group, the preoperative LCEA, ACEA, AAVA, AASA, and PASA were $8^{\circ} \pm 4^{\circ}$, $32^{\circ} \pm 5^{\circ}, 22^{\circ} \pm 2^{\circ}, 42^{\circ} \pm 4^{\circ}$, and $88^{\circ} \pm 2^{\circ}$, respectively, which were corrected to $30^{\circ} \pm 2^{\circ}, 54^{\circ} \pm 4^{\circ}, 19^{\circ} \pm 1^{\circ}$, $58^{\circ} \pm 3^{\circ}$, and $85^{\circ} \pm 2^{\circ}$, respectively, in the virtual PAO The final values of these angles after the actual PAO were $31^{\circ} \pm 3^{\circ}, 56^{\circ} \pm 7^{\circ}, 20^{\circ} \pm 3^{\circ}, 59^{\circ} \pm 5^{\circ}$, and $81^{\circ} \pm 3^{\circ}$, respectively. The deviations between the angles were $7^{\circ} \pm 2^{\circ}, 10^{\circ} \pm 4^{\circ}, 13^{\circ} \pm 8^{\circ}, 8^{\circ} \pm 2^{\circ}$, and $6^{\circ} \pm 2^{\circ}$, respectively. The differences in the deviations between the new developed PAO group and the conventional PAO group, with the exception of the LCEA, were statistically significant $(p<0.05)$ (Table 3$)$.

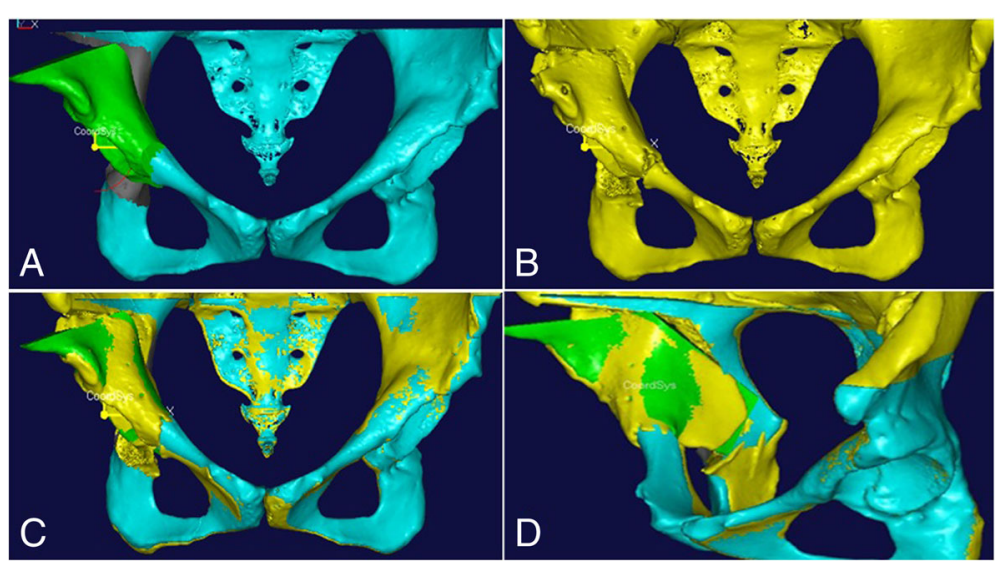

Fig. 6 The reconstructed 3D model of the postoperative pelvis using the new developed PAO was superimposed with the one after virtual PAO. $\mathbf{a}, \mathbf{b}$ The $3 \mathrm{D}$ model of the pelvis after virtual PAO and actual PAO. c, $\mathbf{d}$ The acetabular fragment produced in actual PAO was almost as same as the planned one, and the final position of the acetabular fragment was highly consistent with that planned preoperatively 
Table 3 The radiographic assessment of pre and post PAO

\begin{tabular}{|c|c|c|c|c|c|}
\hline & LCEA & ACEA & AAVA & AASA & PASA \\
\hline \multicolumn{6}{|l|}{ New developed PAO } \\
\hline Preoperative $\left(^{\circ}\right)$ & $10 \pm 5$ & $33 \pm 7$ & $23 \pm 2$ & $42 \pm 4$ & $88 \pm 3$ \\
\hline Planned $\left({ }^{\circ}\right)$ & $30 \pm 2$ & $53 \pm 3$ & $20 \pm 1$ & $59 \pm 2$ & $85 \pm 3$ \\
\hline Postoperative $\left(^{\circ}\right)$ & $31 \pm 2$ & $54 \pm 2$ & $19 \pm 1$ & $58 \pm 2$ & $85 \pm 3$ \\
\hline Deviation (\%) & $4 \pm 1$ & $3 \pm 3$ & $4 \pm 3$ & $3 \pm 2$ & $1 \pm 1$ \\
\hline \multicolumn{6}{|l|}{ Conventional PAO } \\
\hline Preoperative $\left(^{\circ}\right)$ & $8 \pm 4$ & $32 \pm 5$ & $22 \pm 2$ & $42 \pm 4$ & $88 \pm 2$ \\
\hline Planned $\left(^{\circ}\right)$ & $30 \pm 2$ & $54 \pm 4$ & $19 \pm 1$ & $58 \pm 3$ & $85 \pm 2$ \\
\hline Postoperative $\left(^{\circ}\right)$ & $31 \pm 3$ & $56 \pm 7$ & $20 \pm 3$ & $59 \pm 5$ & $81 \pm 3$ \\
\hline Deviation (\%) & $7 \pm 2$ & $10 \pm 4^{*}$ & $13 \pm 8^{*}$ & $8 \pm 2 *$ & $6 \pm 2^{*}$ \\
\hline
\end{tabular}

Values are expressed as mean \pm standard deviation. The deviation between planned value and postoperative value was calculated as the Formula: Deviation $(\%)=\mid$ (postoperative value - planned value)/planed value $\mid \times 100$. ${ }^{*} p<0.01$ compared with values in the new developed PAO group

\section{Discussion}

Bernese periacetabular osteotomy has been used in the treatment of residual hip dysplasia in adolescents and adults for more than 30 years [27, 28]. The amount of angular correction depends on the severity of preoperative dysplasia and varies considerably among patients. Surgeons can plan optimal, individualized operations before surgery, but how to implement it during the actual procedure remains a challenge, which is precisely the primary point for PAO [17, 18]. As insufficient correction of the acetabulum will lead to residual dysplasia, while over-correction in any dimension can cause femoroacetabular impingement $[29,30]$. In addition, while $\mathrm{PAO}$ is a technological empirical procedure that is based on surgeon's clinical experience, the operation itself cannot be completed under direct vision and requires a significant learning curve, which is a burden in the training of new orthopedic specialists.

In this study, we designed and fabricated a customized cutting and rotating template using 3D printing technology and applied it in the actual PAO. The customized cutting template served as a guide to help surgeons slide the osteotome into the precise location that had been determined prior to surgery without the assistance of an image intensifier during PAO. In addition, because of the specificity of the osteotomy site and direction, the possibility of serious osteotomized complications, such as posterior column splitting and/or intraarticular osteotomy, was eliminated. Obtaining the same acetabular fragment that was planned preoperatively is just the first step. Correcting the acetabular fragment to its planned position is the key to a successful PAO. The correction of the acetabular fragment in conventional PAO largely depends on the surgeon's experience and intraoperative X-rays. However, this usually results in greater deviations due to the limitation of the incision length, the patient's position and the surgeon's imprecise subjective observations. Therefore, surgeons need to adjust the location of the acetabular fragment and repeatedly verify the location with an image intensifier. By contrast, the new developed PAO technique allows the fragment to be corrected to the preplanned position through one-off manipulation with the help of a customized rotating template that is embedded into the gap between the fragment and the residual pelvis. Due to its irregular contour, this customized rotating template can be used to precisely position the acetabular fragment at a unique location in the 3D space. Therefore, in our study, the final position of the acetabular fragment was highly consistent with the preoperative planned position, and the postoperative morphological parameters were consistent with the preoperative planned data in the new developed PAO group. Meanwhile, the deviations between the planned and postoperative acetabular angles in the new developed PAO group were less than those of the conventional PAO group $(p<0.05)$. These findings demonstrates that the new developed PAO technique, which uses a customized template, improves the accurate implementation of preoperative planning during the actual operation.

The postoperative record demonstrated that the new developed PAO could not only shorten the duration of the operation but also reduce the number of irradiations used, which benefits both the surgeon and the patient. Due to unskilled installation of the cutting template, the dissection scope of the soft tissue of the acetabular medial wall was larger in the first few cases, which caused the bleeding volume in the new developed PAO group to increase slightly compared with the conventional PAO group. As the techniques became more sophisticated, the bleeding volume of the new developed PAO group subjects decreased compared to the conventional PAO group. This may be because the operation was shorter and surgeons did not have to repeatedly adjust the location of the acetabular fragment with the help of customized template during the operation.

There are several limitations in the current study. First, during the preoperative planning period, we performed idealized processing by designating the femoral head center as the center of rotation and assumed the center of rotation to be fixed. However, to increase the femoral offset and abductor lever arm, the rotation center is usually transferred slightly inferiorly and medially in actual operations. The selected cases were all Crowe I or Crowe II, in which the femoral head is still located in the true acetabulum compared with subluxation or dislocation patients. Therefore, we assumed that the femoral head center could be considered the center of rotation. In addition, the displacement distance of the rotation center during the actual operation is very small 
relative to the femoral head radius. As a result, the impact on experimental error can be ignored. Second, the approximate cost of the customized template was RMB 1000 , and several days are needed to design and fabricate the customized template. In addition, the software is relatively complicated to operate, and some training is needed to develop proficiency. Third, the greatest limitations were the small sample size and the short follow-up. Therefore, it should not jump to a conclusion that the new developed PAO using the customized cutting and rotating template has obvious advantages for the treatment of DDH compared with traditional PAO. However, we are still on the learning curve. By increasing number of patients in the study and extending the follow-up period, we believe that the efficiency of the procedure will be further demonstrated.

\section{Conclusions}

In conclusion, this study demonstrates that our system, which is based on CAD-RP technology, is feasible and could realize the predicted results accurately during the actual PAO. The operation efficiency will further improve along with proficiency with the procedure. In the future, we plan to partner with a commercial organization to develop a more precise and convenient patient-specific osteotomy template, and we expect that it will standardize the PAO for DDH patients.

\section{Abbreviations}

3D: Three-dimensional; CAD: Computer-aided design; DDH: Developmental dysplasia of the hip; HHS: Harris hip score; PAO: Bernese periacetabular osteotomy; RP: Rapid prototyping; VAS: Visual analogue scale

\section{Acknowledgements}

The study was financially supported by the Key Project of Education Department of Anhui Province (KJ2018A0229) and Science and Technology Development Fund of Bengbu Medical College (BYKF1736).

\section{Authors' contributions \\ XW and SL participated in the design of the study, manuscript preparation, data statistical analysis, and revision. JP advised and assisted in the drafting of the manuscript. ZZ participated in revising critically the manuscript. LZ advised and assisted in the design and fabrication of the customized cutting and rotating template. JG and XC is responsible for study design, surgical operation, and general supervision of the research group. All authors read and approved the final manuscript.}

\section{Funding}

Not applicable

\section{Availability of data and materials}

All relevant raw data for this study have been presented in the main manuscript or additional supporting files, which are freely available to any scientist wishing to use them, without breaching participant confidentiality.

\section{Ethics approval and consent to participate}

All investigations were conducted in conformity with the Helsinki Declaration and the study protocol was approved by our institutional review board (Ethics Committee of Xin Hua Hospital Affiliated to Shanghai Jiao Tong University School of Medicine, Approval No, XHEC-D-2016-008). All patients received a thorough explanation of this study and gave their oral and written informed consent to be included in this analysis.

\section{Consent for publication}

Consent for publication was obtained from all patients.

\section{Competing interests}

The authors declare that they have no competing interests.

\section{Author details}

${ }^{1}$ Department of Orthopaedics, The First Affiliated Hospital of Bengbu Medical College, Bengbu, Anhui, China. ²Department of Orthopaedics, Xinhua Hospital affiliated to Shanghai Jiaotong University School of Medicine, Shanghai, China. ${ }^{3}$ The First Affiliated Hospital of Bengbu Medical College, Bengbu, Anhui, China. ${ }^{4}$ Department of Biomedical Engineering, Shanghai University Of Technology, Shanghai, China.

Received: 29 March 2019 Accepted: 8 July 2019

Published online: 16 July 2019

\section{References}

1. Peters CL, Erickson JA, Anderson L, Anderson AA, Weiss J. Hip-preserving surgery: understanding complex pathomorphology. J Bone Joint Surg Am. 2009;91(Suppl 6):42-58.

2. Li PL, Ganz R. Morphologic features of congenital acetabular dysplasia: one in six is retroverted. Clin Orthop Relat Res. 2003;416:245-53.

3. Ito H, Matsuno T, Hirayama T, Tanino H, Yamanaka Y, Minami A. Three-dimensional computed tomography analysis of non-osteoarthritic adult acetabular dysplasia. Skelet Radiol. 2009;38(2):131-9.

4. Panagiotopoulou N, Bitar K, Hart WJ. The association between mode of delivery and developmental dysplasia of the hip in breech infants: a systematic review of 9 cohort studies. Acta Orthop Belg. 2012;78(6):697-702.

5. Beck M, Kalhor M, Leunig M, Ganz R. Hip morphology influences the pattern of damage to the acetabular cartilage: femoroacetabular impingement as a cause of early osteoarthritis of the hip. J Bone Joint Surg Br. 2005;87(7):1012-8.

6. Ganz R, Parvizi J, Beck M, Leunig M, Notzli H, Siebenrock KA. Femoroacetabular impingement: a cause for osteoarthritis of the hip. Clin Orthop Relat Res. 2003;417:112-20.

7. Tanzer M, Noiseux N. Osseous abnormalities and early osteoarthritis: the role of hip impingement. Clin Orthop Relat Res. 2004;429:170-7.

8. Naito M, Nakamura Y. Curved periacetabular osteotomy for the treatment of dysplastic hips. Clin Orthop Surg. 2014;6(2):127-37.

9. Tonnis D, Behrens K, Tscharani F. A new technique of triple osteotomy for turning dysplastic acetabula in adolescents and adults (author's transl). Z Orthop Ihre Grenzgeb. 1981;119(3):253-65.

10. Ninomiya S, Tagawa H. Rotational acetabular osteotomy for the dysplastic hip. J Bone Joint Surg Am. 1984;66(3):430-6.

11. Ganz R, Klaue K, Vinh TS, Mast JW. A new periacetabular osteotomy for the treatment of hip dysplasias. Technique and preliminary results. Clin Orthop Relat Res. 1988;232:26-36.

12. Steppacher SD, Tannast M, Ganz R, Siebenrock KA. Mean 20-year followup of Bernese periacetabular osteotomy. Clin Orthop Relat Res. 2008;466(7):1633-44.

13. Kralj M, Mavcic $B$, Antolic $V$, Iglic $A$, Kralj-lglic $V$. The Bernese periacetabular osteotomy: clinical, radiographic and mechanical 7-15-year follow-up of 26 hips. Acta Orthop. 2005;76(6):833-40.

14. Zhu J, Chen X, Cui Y, Shen C, Cai G. Mid-term results of Bernese periacetabular osteotomy for developmental dysplasia of hip in middle aged patients. Int Orthop. 2013;37(4):589-94.

15. Wang X, Peng J, Zhu J, Shen C, Cui Y, Chen X. Application of threedimensional computerised tomography reconstruction and image processing technology in individual operation design of developmental dysplasia of the hip patients. Int Orthop. 2016;40(2):255-65.

16. Saragaglia D, Massfelder J, Refaie R, Rubens-Duval B, Mader R, Rouchy RC, Pailhe R. Computer-assisted total knee replacement after medial opening wedge high tibial osteotomy: medium-term results in a series of ninety cases. Int Orthop. 2016;40(1):35-40.

17. Zou Z, Chavez-Arreola A, Mandal P, Board TN, Alonso-Rasgado T. Optimization of the position of the acetabulum in a ganz periacetabular osteotomy by finite element analysis. J Orthop Res. 2013;31(3):472-9.

18. Zhao X, Chosa E, Totoribe K, Deng G. Effect of periacetabular osteotomy for acetabular dysplasia clarified by three-dimensional finite element analysis. J Orthop Sci. 2010;15(5):632-40. 
19. Akiyama H, Goto K, So K, Nakamura T. Computed tomography-based navigation for curved periacetabular osteotomy. J Orthop Sci. 2010; 15(6):829-33.

20. Hsieh PH, Chang YH, Shih CH. Image-guided periacetabular osteotomy: computer-assisted navigation compared with the conventional technique: a randomized study of 36 patients followed for 2 years. Acta Orthop. 2006; 77(4):591-7.

21. Wang $X$, Peng J, Li, Zhang L, Wang $H$, Jiang $L$, Chen $X$. Does the optimal position of the acetabular fragment should be within the radiological normal range for all developmental dysplasia of the hip? A patient-specific finite element analysis. J Orthop Surg Res. 2016;1 1(1):109.

22. Otsuki B, Takemoto M, Kawanabe K, Awa Y, Akiyama H, Fujibayashi S, Nakamura T, Matsuda S. Developing a novel custom cutting guide for curved peri-acetabular osteotomy. Int Orthop. 2013;37(6):1033-8.

23. Omori S, Murase T, Kataoka T, Kawanishi Y, Oura K, Miyake J, Tanaka H, Yoshikawa H. Three-dimensional corrective osteotomy using a patientspecific osteotomy guide and bone plate based on a computer simulation system: accuracy analysis in a cadaver study. Int J Med Robot. 2014;10(2): 196-202.

24. Won SH, Lee YK, Ha YC, Suh YS, Koo KH. Improving pre-operative planning for complex total hip replacement with a rapid prototype model enabling surgical simulation. Bone Joint J. 2013:95-B(11):1458-63.

25. Crowe JF, Mani VJ, Ranawat CS. Total hip replacement in congenital dislocation and dysplasia of the hip. J Bone Joint Surg Am. 1979;61 (1):15-23.

26. Benum P, Aamodt A, Nordsletten L. Customised femoral stems in osteopetrosis and the development of a guiding system for the preparation of an intramedullary cavity: a report of two cases. J Bone Joint Surg Br. 2010;92(9):1303-5.

27. Garras DN, Crowder TT, Olson SA. Medium-term results of the Bernese periacetabular osteotomy in the treatment of symptomatic developmental dysplasia of the hip. J Bone Joint Surg Br. 2007;89(6):721-4.

28. Siebenrock KA, Schaller C, Tannast M, Keel M, Buchler L. Anteverting periacetabular osteotomy for symptomatic acetabular retroversion: results at ten years. J Bone Joint Surg Am. 2014;96(21):1785-92.

29. Myers SR, Eijer H, Ganz R. Anterior femoroacetabular impingement after periacetabular osteotomy. Clin Orthop Relat Res. 1999;363:93-9.

30. Yasunaga Y, Yamasaki T, Matsuo T, Ishikawa M, Adachi N, Ochi M. Crossover sign after rotational acetabular osteotomy for dysplasia of the hip. J Orthop Sci. 2010;15(4):463-9.

\section{Publisher's Note}

Springer Nature remains neutral with regard to jurisdictional claims in published maps and institutional affiliations.

Ready to submit your research? Choose BMC and benefit from:

- fast, convenient online submission

- thorough peer review by experienced researchers in your field

- rapid publication on acceptance

- support for research data, including large and complex data types

- gold Open Access which fosters wider collaboration and increased citations

- maximum visibility for your research: over $100 \mathrm{M}$ website views per year

At $\mathrm{BMC}$, research is always in progress.

Learn more biomedcentral.com/submissions 